\title{
Influence of heat radiation of high intensity on the temperature of thermally thin body
}

\author{
Dana Baktybaeva ${ }^{1}$, Timur Baygushkarov ${ }^{1}$, and Vyacheslav Yukhnov ${ }^{1}$ \\ ${ }^{1}$ Tomsk Polytechnic University, Department of Theoretical and Industrial Heat System Engineering, \\ 634050 Tomsk, Russia
}

\begin{abstract}
It is established that the source with the heat radiance high density influence on the temperature of the thermally thin sample three times greater when using a metallic screen instead of the water curtain. Therefore, the decrease in intensive heat transfer heat (or burning) is the most effective water curtain.
\end{abstract}

The relevance of studies of thermal radiation associated with hazardous processes, such as in the chemical industry for the organization of high-temperature processes in the open air with a system of thermal protection in the form of a water curtain. The published results of experimental studies of regularities of the processes of heat and mass occurring during the movement of a single or groups of drops in the high-temperature gaseous medium can not be used in the analysis of heat transfer processes at the water curtains. The research results of a single drop of water or a group differ significantly from the characteristic dimensions of the applied water curtains, so it is appropriate experimental study of the laws of heat transfer processes in intensive heat (or burning) with a water curtain.

Therefore, to obtain reliable experimental data in the study of heat sources, open flame of the surrounding objects is an important practical task. The aim of the present work is an experimental studying of an influence of distance from the source with the heat radiance density $55.103 \mathrm{~W} / \mathrm{m} 2$ on studying thermally thin sample.

The objectives of the study are to measure the temperature of the sample from different distances of the source of thermal radiation, as well as the definition of the analytical dependence of the temperature of the sample at different distances from the source of thermal radiation.

A scheme of the experimental setup area on which the research was conducted in accordance with Fig. 1. The installation consists of a source of thermal radiation $\left(T_{1}\right)$, sample temperature sensor $\left(T_{2}\right)$, replaceable screen (metal screen or water curtain), and measuring the distance from the source of thermal radiation to the sample with temperature sensor.

A source of thermal radiation was the flame of the burner, placed in the flame of a thermoelectric transducer for temperature measurement. The distance $\mathrm{x}$ between the source of thermal radiation and the irradiated sample varies from $8 \cdot 10^{-2} \mathrm{~m}$. to $20 \cdot 10^{-2} \mathrm{~m}$. rates. The Measurement of the steady-state temperature of the sample $T_{2}$ was conducted at an average temperature of the source of thermal radiation is equal $993,15 \mathrm{~K}$. The ambient temperature was maintained constant equal $295,15 \mathrm{~K}$.

This is an Open Access article distributed under the terms of the Creative Commons Attribution License 4.0, which permits unrestricted use, distribution, and reproduction in any medium, provided the original work is properly cited. 


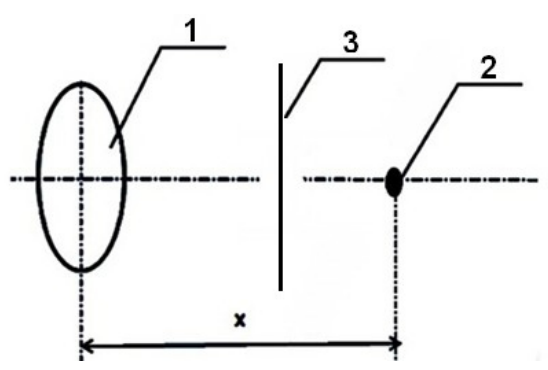

Figure 1. The measuring scheme of the experimental setup: 1- the radiation source, 2 - the sample, 3 - metal screen or water curtain, $x$ - the distance from the radiation source to the sample.

Each measurement was performed three times. The results of statistical data processing with regard to the detection and elimination of gross errors (blunders) obtained in accordance with Fig. 2.

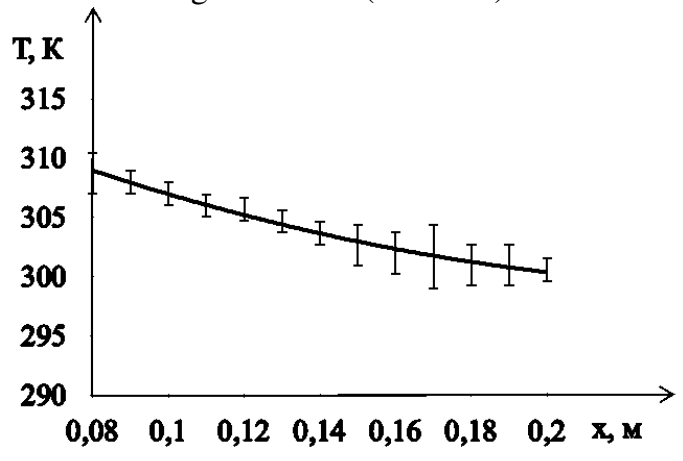

Figure 2. Experimental data of the temperature of the sample $T_{2}$ at different distances $\mathrm{x}$ from the radiation source when metal screen: 1 - graph of average temperature (1), 2 - experimental data.

In accordance with Fig. 2, the results showed that with increasing distance $x$ between the source of thermal radiation and the sample, the temperature decreases. The experimental data of the temperature of the sample, located at different distances from the source of thermal radiation, in accordance with Fig. 2, satisfactorily describes the approximate dependence represents a polynomial function

$$
T(x)=a_{0}+a_{1} x+a_{2} x^{2}, \mathrm{~K},
$$

where the coefficients $a_{0}=319,15, a_{1}=-147,8, a_{2}=274,73$. The approximation error does not exceed $0,5 \%$ rate.

The experimental data shown in Fig. 3, showed that the temperature of the sample is reduced by using a water curtain. The experimental data of the temperature of the sample, located at different distances from the source of thermal radiation and separated water curtain, in accordance with Fig. 3, satisfactorily describes the approximate dependence (1) where the coefficients $a_{0}=319,15$, $a_{1}=-147,8, a_{2}=274,73$. The approximation error does not exceed $0,5 \%$ rate. 
TSOTR 2015

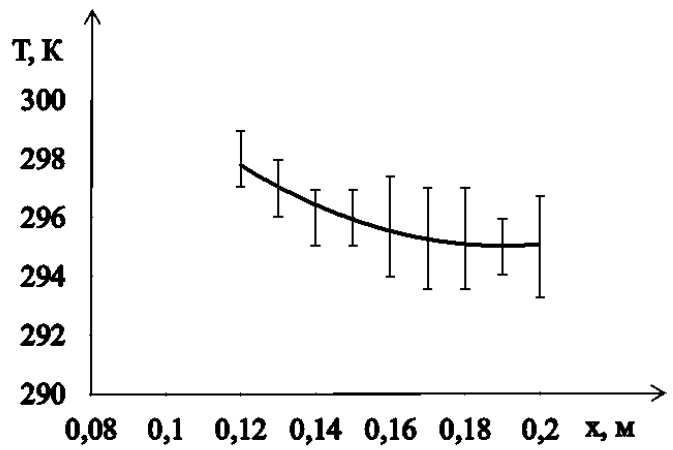

Figure 3. Graphs of temperature change of the sample $T_{2}$ the distance $x$ when water curtain: 1 - graph of average temperature (1), 2 - experimental data.

The heat flux density on the surface of the sample depends on the distance $\mathrm{x}$ from the source of thermal radiation. This relationship is described by the equation

$$
E_{2}(x)=b_{0}+b_{1} x+b_{2} x^{2}+b_{3} x^{3}, \mathrm{~K},
$$

where the coefficients $b_{0}=7,2804, b_{1}=-93,089, b_{2}=441,48, b_{3}=-736,53$. Equation (2) suitable for determining the mean density of heat flux on the sample surface at a distance of $12 \cdot 10^{-2} \leq x \leq$ $20 \cdot 10^{-2} \mathrm{~m}$. from the source of thermal radiation with an error not exceeding $0,5 \%$.

Conclusion. The study and experimental data on the dependence of the temperature of the irradiated sample distance $x$ to the source of thermal radiation, in the range from $8 \cdot 10^{-2} \mathrm{~m}$. to $20 \cdot 10^{-2}$ $\mathrm{m}$. with a source temperature of thermal radiation equal $993,15 \mathrm{~K}$. It is established that the source with the heat radiance density $55 \cdot 10^{3} \mathrm{~W} / \mathrm{m}^{2}$ influence on the temperature of the thermally thin sample three times greater when using a metallic screen instead of the water curtain. Increasing the distance between the source and the sample to $20 \cdot 10^{-2} \mathrm{~m}$ by using a metal screen to reduce the temperature of the thermally thin sample of $3 \%$. If the radiation source is installed in front of the water curtain, the thin sample temperature thermally weakly dependent on the distance $\mathrm{x}$, and the change in temperature of the sample is less than $1 \%$. Therefore, the water curtain is the most effective way to decrease heat transfer in systems of intense heat (or burning). 